ORIGINAL BREVE

\title{
PREVALENCIA DE LA ENFERMEDAD RENAL CRÓNICA DETERMINADA MEDIANTE LA APLICACIÓN DE ECUACIONES PREDICTIVAS EN PERSONAS HIPERTENSAS ATENDIDAS EN ATENCION PRIMARIA
}

\author{
Rafael Gómez Navarro \\ Equipo de Atención Primaria Teruel Rural. Consejería de Sanidad de Aragón.
}

\section{RESUMEN}

Fundamento: La enfermedad renal crónica (ERC) constituye un importante problema de salud en las sociedades desarrolladas vinculado al progresivo envejecimiento de la población y a la elevada prevalencia de patologías como la hipertensión arterial (HTA) y la diabetes mellitus. Los objetivos de este trabajo son: estudiar la función renal (FR) en las personas hipertensas mediante ecuaciones predictivas y creatinina plasmática (Crp). Conocer el porcentaje de personas con enfermedad renal crónica (ERC) que presentan valores normales de Crp. Analizar los factores que colaboran en el deterioro de su FR.

Metodos: Estudio descriptivo transversal de las personas con hipertensión arterial (HTA). Se determinó Crp y tensión arterial (TA). Se calculó el filtrado glomerular mediante las fórmulas de Cockroft-Gault y MDRD. Se registraron los años de evolución de la HTA. Se realizó estudio descriptivo de las variables estudiadas y se analizó la posible dependencia entre algunas mediante regresión lineal múltiple..

Resultados: Se estudió un total de 52 pacientes $(57,7 \%$ mujeres). Edad media 72,4 $\pm 10,8$. Un 32,6\% (Cockcroft-Gault) o un $21,5 \%$ (MDRD) cumplían criterio de ERC. Predomina la ERC en las mujeres. El 21,4\% (Cockcroft-Gault) y 9,5\% (MDRD) de pacientes con ERC tenían valores normales de Crp. No encontramos dependencia lineal entre las cifras de TA y la FR. El cumplimiento de los objetivos de TA no supone un menor desarrollo de ERC. En los varones encontramos dependencia lineal entre la FR (MDRD) y los años de evolución de la HTA.

Conclusiones: La ERC es una patología frecuente en las personas hipertensas. La utilización sistemática de ecuaciones predictivas facilita la detección de ERC oculta en pacientes con Crp normal.

Palabras clave: Fallo renal crónico. Creatinina. Ecuación de Cockroft-Gault. Ecuación MDRD. Hipertensión arterial. Atención Primaria.

Correspondencia: C/ Dean Buj, s/n 44001 Teruel ragona@ya.com

\section{ABSTRACT}

\section{Prevalence of Chronic Kidney Disease in Hypertensive Persons Attended in Primary Care from Spain Determined by Application of Estimating Equations}

Background: To study the renal function (FR) of the hypertensive patients by means of estimating equations and serum creatinine (Crp). To calculate the percentage of patients with chronic kidney disease (ERC) that present normal values of Crp. To analyze which factors collaborate in the deterioration of the FR.

Methods: Descriptive cross-sectional study of patients with HTA. Crp and arterial tension (TA) were determined. The glomerular filtration rate was calculated by means of Cockroft-Gault and MDRD's formula. The years of evolution of the HTA were registered. A descriptive study of the variables and the possible dependence among them was completed, using several times linear multiple regression.

Results: 52 patients were studied $(57,7 \%$ women). Average age $72,4 \pm 10,8.32,6 \%$ (Cockcroft-Gault) or 21,5\% (MDRD) were fulfilling ERC criterion. The ERC was mainly diagnosed in females. $21,4 \%$ (Cockcroft-Gault) and 9,5\% patients (MDRD) with ERC had normal Crp values. We do not find linear dependence between the numbers of TA and the FR. The TA check-up objectives do not suppose less development of ERC. In males we find linear dependence within the FR (MDRD) and the years of evolution of the HTA.

Conclusion: The ERC is a frequent pathology in the hypertense persons. The systematical utilization of estimating equations facilitates the detection of hidden ERC in patients with normal Crp.

Key words: Kidney Failure Chronic. Creatinine. Hypertension. Primary health care. Spain. 


\section{INTRODUCCION}

Definimos la enfermedad renal crónica (ERC) como aquélla en la que existe una disminución de la función renal expresada por un filtrado glomerular (FG) o por un aclaramiento de creatinina $(\mathrm{ClCr})$ estimado inferior a $60 \mathrm{ml} / \mathrm{min} / 1,73 \mathrm{~m}^{2}$ o como la presencia de daño renal persistente durante al menos 3 meses $^{1}$.

La ERC constituye hoy en día un importante problema de salud en las sociedades desarrolladas, indudablemente vinculado al progresivo envejecimiento de la población y a la elevada prevalencia de patologías como la hipertensión arterial (HTA) y la diabetes mellitus.

El estudio poblacional americano NHANES III $^{2}$ destaca que el $4,7 \%$ de la población mayor de 20 años presenta un $\mathrm{ClCr}$ inferior a $60 \mathrm{ml} / \mathrm{min} / 1,73 \mathrm{~m}^{2}$. En nuestro país Otero y cols. ${ }^{3}$ y Simal y cols. ${ }^{4}$ cifran esta prevalencia en el $13 \%$ y $8 \%$ respectivamente, llegando hasta el $49 \%$ en población general mayor de 64 años ${ }^{5}$.

Se ha objetivado que el riesgo relativo de padecer nefropatía crónica cuando existe HTA aislada es de $1,57^{6}$. La mayoría de los estudios apoyan una posible relación entre los niveles de tensión arterial y la aparición de insuficiencia renal ${ }^{7}$.

La ERC en la mayoría de los casos es en sus estadíos iniciales asintomática. Habitualmente su detección se produce en los controles analíticos rutinarios con determinación de la creatinina plasmática (Crp) que en atención primaria se realizan periódicamente a los pacientes hipertensos. Sin embargo, es conveniente destacar que los valores de Crp pueden permanecer normales incluso en pacientes que presentan una función renal muy deteriorada ${ }^{8,9}$.

Para facilitar la correcta identificación y el diagnóstico precoz en estos pacientes se propugna la utilización sistemática de fórmulas o ecuaciones predictivas y de fácil aplicación en atención primaria como son la de Cockroft-Gault ${ }^{10}$ y MDRD abreviada (Modification of diet in Renal Disease) ${ }^{11}$.

Los objetivos de este trabajo son: determinar y estadiar la función renal de las personas hipertensas mediante ecuaciones predictivas y Crp. Determinar el porcentaje de pacientes con deterioro de la función renal que se ocultan tras valores normales de Crp $\mathrm{y}$ analizar los distintos factores que pueden colaborar en el deterioro de la función renal de las personas con HTA.

\section{SUJETOS Y MÉTODOS}

Estudio descriptivo transversal realizado entre el 26 de Octubre de 2007 y el 15 de Marzo de 2008 en Caudé, Teruel, que en el momento de comenzar el estudio contaba con una población de referencia de 265 habitantes.

La población en estudio fueron las personas diagnosticadas de HTA. Se estudió todo el censo de la población diana, no procediendo por lo tanto ningún tipo de muestreo.

Se consideraron como causa de excepción aquellas circunstancias en las que no es adecuado determinar la función renal mediante ecuaciones predictivas: peso corporal extremo (IMC $<19 \mathrm{~kg} / \mathrm{m}^{2}$ o $>35$ $\mathrm{kg} / \mathrm{m}^{2}$ ); alteraciones importantes de la masa muscular (amputaciones, pérdida de masa muscular, enfermedades musculares o parálisis); insuficiencia renal aguda; embarazo con hepatopatía grave y edema generalizado o ascitis.

A todas las personas se les solicitó Crp, se les midió su tensión arterial y se estimó su FG mediante las fórmulas de CockroftGault $^{10}$ y MDRD abreviada ${ }^{11}$. 


$$
\begin{aligned}
F G= & 186 \times \text { Creatinina sérica }(\mathrm{mg} / \mathrm{dl})^{-1,154} \times(\text { edad })^{-0,203} \\
& x(0,742 \text { si es mujer }) \times(1,21 \text { si raza negra }) \\
C C r= & \frac{(140 \text {-edad }[\text { años] }) \times \text { peso }(\mathrm{kg})}{\text { Creatinina sérica }(\mathrm{mg} / \mathrm{dl}) \times 72}(x 0,85 \text { en mujeres })
\end{aligned}
$$

Se recomienda ofrecer los datos corregidos por $1,73 \mathrm{~m}^{2}$ de superficie corporal

Cálculo de la superficie corporal: Fórmula de DuBois:

Superficie corporal $\left(\mathrm{m}^{2}\right)=0,20247 \times$ altura $(\mathrm{m})^{0,725}$ x peso $(\mathrm{kg})^{0,425}$

La interpretación y estadiaje del resultado del FG se llevó a cabo según la Guía de la National Kidney Foundatión (NKF) ${ }^{12}$.

\begin{tabular}{|c|l|c|}
\hline Estadío & \multicolumn{1}{|c|}{ Descripción } & FG \\
\hline 1 & Daño renal* con TFG normal & $\geq 90$ \\
\hline 2 & $\begin{array}{l}\text { Daño renal* con TFG ligeramente } \\
\text { disminuido }\end{array}$ & $60-89$ \\
\hline 3 & FG moderadamente disminuido & $30-59$ \\
\hline 4 & FG gravemente disminuido & $15-29$ \\
\hline 5 & Fallo renal & $<15$ ó diálisis \\
\hline
\end{tabular}

*Daño renal: alteraciones en las pruebas complementarias de orina, sangre o diagnóstico por imágenes.

La posible dependencia lineal entre variables cuantitativas se estudió mediante modelos de regresión lineal múltiple. La posible relación entre variables cualitativas se estudió mediante la prueba de chi-cuadrado.

Los datos fueron procesados con el paquete estadístico SPSS 15.0 (SPSS INC, Chicago; IL).

\section{RESULTADOS}

La población diana estuvo formada por un total de 55 personas de las que se excluyeron 3 casos: dos por presentar IMC $>35$ $\mathrm{kg} / \mathrm{m}^{2}$ y una por ascitis en el seno de una hepatopatía grave, ascendiendo el número final de participantes estudiados a 52 (57,7\% mujeres) con edad media 72,4 \pm 10,8. La tabla 1 presenta los resultados obtenidos en cuanto al estadiaje de la función renal obtenido con cada una de las ecuaciones. Con ambas estimaciones determinamos que un 67,3\% (Ecuación de Cockcroft-Gault) o un 78,8\% (ecuación MDRD) de nuestra población presentan un $\mathrm{ClCr}>60$ $\mathrm{ml} / \mathrm{min} / 1,73 \mathrm{~m}^{2}$.

Considerando criterio de ERC presentar un $\mathrm{ClCr}<60 \mathrm{ml} / \mathrm{min} / 1,73 \mathrm{~m}^{2}$ o una $\mathrm{Crp}>$ $1,2 \mathrm{mg} / \mathrm{dl}$, podemos apreciar en la tabla 2 cómo las mayores prevalencias se detectan con la aplicación de la fórmula de CockroftGault. y las más bajas se encuentran si utilizamos la determinación de Crp.

No encontramos en el total de la población dependencia lineal $(\mathrm{p}=0,179)$ entre las cifras de Crp, la edad y los años de evolución de la hipertensión. En el subgrupo de mujeres encontramos que por cada año de edad, la Crp se incrementa en $0,011 \mathrm{mg} / \mathrm{dl}$ y en los varones cada año de evolución de la HTA incrementa la Crp en 0,018 mg/dl.

Al analizar la función renal estudiada mediante la fórmula de Cockroft-Gault en

tabla 1

Estadiaje de los resultados obtenidos mediante ambas ecuaciones

\begin{tabular}{|l|c|c|c|c|c|c|}
\hline & \multicolumn{3}{|c|}{ Ecuación de Cockcroft-Gault } & \multicolumn{3}{c|}{ Ecuación MDRD } \\
\cline { 2 - 7 } & $\begin{array}{c}\text { Total } \\
(\mathbf{n = 5 2})\end{array}$ & $\begin{array}{c}\text { Varones } \\
(\mathbf{n = 2 2})\end{array}$ & $\begin{array}{c}\text { Mujeres } \\
(\mathbf{n = 3 0})\end{array}$ & $\begin{array}{c}\text { Total } \\
(\mathbf{n = 5 2})\end{array}$ & $\begin{array}{c}\text { Varones } \\
(\mathbf{n = 2 2})\end{array}$ & $\begin{array}{c}\text { Mujeres } \\
(\mathbf{n = 3 0})\end{array}$ \\
\hline Estadío 1 & $19,2 \%$ & $31,8 \%$ & $10 \%$ & $9,6 \%$ & $13,6 \%$ & $6,7 \%$ \\
\hline Estadío 2 & $48,1 \%$ & $45,5 \%$ & $50 \%$ & $69,2 \%$ & $77,3 \%$ & $63,3 \%$ \\
\hline Estadío 3 & $30,8 \%$ & $18,2 \%$ & $40 \%$ & $21,2 \%$ & $9,1 \%$ & $30,0 \%$ \\
\hline Estadío 4 & $1,9 \%$ & $4,5 \%$ & $0 \%$ & $0,0 \%$ & $0,0 \%$ & $0,0 \%$ \\
\hline Estadío 5 & $0,0 \%$ & $0,0 \%$ & $0 \%$ & $0,0 \%$ & $0,0 \%$ & $0,0 \%$ \\
\hline
\end{tabular}


Tabla 2

Prevalencia de enfermedad renal crónica obtenida en pacientes hipertensos según el método diagnóstico y de enfermedad renal crónica oculta que ambos métodos hallan, desagregado por sexo

\begin{tabular}{|c|c|c|c|c|c|c|c|c|}
\hline & \multicolumn{2}{|c|}{$\begin{array}{l}\text { Creatinina plasmática } \\
\qquad>1,2 \mathrm{mg} / \mathrm{dl}\end{array}$} & \multicolumn{3}{|c|}{$\begin{array}{c}\mathbf{F G}<60 \mathrm{ml} / \mathrm{min} / \mathbf{1 , 7 3} \mathrm{m}^{2} \\
(\text { Cockroft-Gault) }\end{array}$} & \multicolumn{3}{|c|}{ 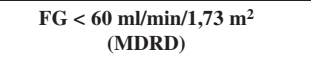 } \\
\hline & $\mathbf{n}$ & $\%$ & $\mathbf{n}$ & $\%$ & $\begin{array}{l}\text { \% ERC } \\
\text { oculta }\end{array}$ & $\mathbf{n}$ & $\%$ & $\begin{array}{c}\text { \% ERC } \\
\text { oculta }\end{array}$ \\
\hline Total $(\mathrm{N}=52)$ & 6 & 11,5 & 17 & 32,6 & 21,1 & 11 & 21,5 & 10 \\
\hline Varones $(\mathrm{N}=22)$ & 3 & 13,6 & 5 & 22,7 & 9,1 & 2 & 9,0 & $-4,6$ \\
\hline Mujeres $(\mathrm{N}=30)$ & 3 & 10,0 & 12 & 40,0 & 30,0 & 9 & 30,0 & 20 \\
\hline
\end{tabular}

la población global (hombres y mujeres) y la variable edad, encontramos un deterioro de $2,121 \mathrm{ml} / \mathrm{min} / 1,73 \mathrm{~m}^{2}$ por cada año. Desglosado según sexo encontramos un deterioro anual de $2,576 \mathrm{ml} / \mathrm{min} / 1,73 \mathrm{~m}^{2}$ en las mujeres y de $1,727 \mathrm{ml} / \mathrm{min} / 1,73 \mathrm{~m}^{2}$ en los varones. No hubo dependencia lineal entre los valores arrojados por la fórmula de Cockroft-Gault y la variable años de evolución de la enfermedad.

Realizando el mismo análisis con los resultados obtenidos mediante la fórmula MDRD abreviada hubo un deterioro de la función renal de $0,694 \mathrm{ml} / \mathrm{min} / 1,73 \mathrm{~m}^{2}$ anual en el total de la población, que es de $1,206 \mathrm{ml} / \mathrm{min} / 1,73 \mathrm{~m}^{2}$ en el subgrupo de las mujeres. En el subgrupo de los varones sí encontramos dependencia lineal entre el deterioro de la función renal determinado por esta fórmula y los años de evolución de la HTA, cifrándose en un decremento de $1,539 \mathrm{ml} / \mathrm{min} / 1,73 \mathrm{~m}^{2}$ por cada año.

No hemos encontrado dependencia lineal entre los valores de presión arterial sistólica o presión arterial diastólica y los de Crp ni de función renal determinado por cualquiera de las dos fórmulas, ni en la población general ni en ninguno de los dos subgrupos de género.

\section{DISCUSION}

Aunque la mayoría de los participantes presentan una buena función renal no puede pasar inadvertido que un alto porcentaje de personas hipertensas presentan alteración en su función renal.

El método más elemental para el estudio de la función renal es la determinación de la Crp, pero es bien conocido que al poder verse afectado por diversos factores (edad y masa muscular principalmente) las conclusiones extraídas a partir de este parámetro deben hacerse con ciertas reservas. Especialmente inadecuada es su utilización en pacientes de edad avanzada ya que debido a su habitual escasa masa muscular, valores normales de Crp "ocultan" una insuficiencia renal de mayor o menor entidad ${ }^{17}$. En nuestro caso, con personas hipertensas cuya edad media se sitúa en los 72 años, la fórmula de Cockroft-Gault y la MDRD abreviada nos permitieron identificar a un $21,1 \%$ y $10 \%$ respectivamente de pacientes con ERC que hubieran permanecido sin diagnosticar por presentar valores normales de $\operatorname{Crp}(<1,2 \mathrm{mg} / \mathrm{dl})$. . Es en el subgrupo de las mujeres donde encontramos más altas prevalencias de ERC, llegando a triplicar y cuadruplicar sus cifras al utilizar las fórmulas MDRD y de Cockroft-Gault respectivamente a las que encontráramos si utilizáramos exclusivamente la Crp.

El estudio de Buitrago $\mathrm{F}$ et al. ${ }^{13}$ realizado con una cohorte de 845 pacientes de los que el $79,4 \%$ eran hipertensos y considerando función renal normal a valores de Crp $<1,3 \mathrm{mg} / \mathrm{dl}$ en mujeres y $\mathrm{Crp}<1,4 \mathrm{mg} / \mathrm{dl}$ en varones hallaron un 8,3\% de ERC oculta utilizando la fórmula de Cockroft-Gault y un $11,6 \%$ mediante la MDRD. El estudio 
EROCAP ${ }^{14}$ realizado en una amplia muestra compuesta por 9.223 pacientes de atención primaria, de los que el $66,7 \%$ estaban diagnosticados de HTA, encontró una prevalencia de ERC oculta del 7,9\% utilizando la fórmula MDRD considerando valores normales de $\mathrm{Crp}<1,1 \mathrm{mg} / \mathrm{dl}$ en mujeres y $\mathrm{Crp}<1,2 \mathrm{mg} / \mathrm{dl}$ en varones. Observamos que la prevalencia de ERC oculta encontrada en nuestra población compuesta sólo por pacientes hipertensos utilizando la fórmula MDRD es muy similar a la de estos estudios y claramente superior si el diagnóstico lo realizamos utilizando la de CockroftGault.

Específicamente diseñado para determinar ERC en pacientes hipertensos es el trabajo de Pozuelos G et al. ${ }^{15}$ que estudiando 113 pacientes con una media de edad muy similar a la nuestra, encuentran una prevalencia empleando la fórmula MDRD del $27 \%$, ligeramente superior a la nuestra.

El recientemente publicado Documento de consenso sobre la enfermedad renal crónica $\mathbf{1 6}$ elaborado conjuntamente por la Sociedad Española de Medicina de Familia y Comunitaria y la Sociedad Española de Nefrología establece una serie de recomendaciones claras sobre el manejo de los pacientes con ERC en nuestro nivel asistencial y promueve el uso sistemático de ecuaciones predictivas para el cálculo del FG, indicando como primera opción la MDRD y como alternativa válida la de CockroftGault. La generalización de esta práctica pensamos que sería un buen mecanismo para conseguir un mejor diagnóstico de la ERC en atención primaria.

Olivares J et al, ${ }^{17}$ en un estudio realizado sobre 2.249 hipertensos encontraron que el 40,4\% tenían $\mathrm{ClCr}<60 \mathrm{ml} . / \mathrm{min}$. pero no detectaron asociación de este decremento del FG con las cifras tensionales. Son pues resultados claramente peores en cuanto a prevalencia de ERC que los que tenemos en nuestra población y como nosotros tampo- co encuentran asociación entre el deterioro de la función renal y los valores de presión arterial.

Con nuestros datos, la hipótesis de que los participantes que cumplen los objetivos terapéuticos en HTA desarrollan menos ERC (determinada por cualquiera de las tres maneras empleadas) no puede ser aceptada ni rechazada en nuestra población general. Sin embargo, y desde un punto de vista cualitativo encontramos que no más de $1 / 3$ de los pacientes que cumplen los objetivos terapéuticos desarrollan.

La razón de esto es sin duda el pequeño tamaño de la muestra y nos parece innecesario insistir en la conveniencia de tratar de cumplir los objetivos de control tensional.

El género ha influido de manera significativa en nuestros resultados. Aunque los valores medios de FG son mejores en los varones que en las mujeres, éstas predominan claramente entre los pacientes incluidos en el estadio 3. Este hallazgo es similar al Estudio Hortega ${ }^{\mathbf{1 8}}$ en el que casi la mitad de las mujeres mayores de 65 años padecían enfermedad renal crónica estadio 3 , frente a sólo un tercio de los varones.

Nuestro estudio presenta algunas limitaciones importantes que debemos señalar. El primero de ellos sin ninguna duda es el escaso número de pacientes estudiados, que si bien son todas las personas hipertensas de nuestra población, su limitado número condiciona el análisis estadístico y dificulta la extracción de conclusiones válidas y extrapolación de nuestros resultados. Creemos que este hecho viene compensado por la circunstancia de que no hemos encontrado en la bibliografía española ningún trabajo similar realizado en el medio rural.

Tampoco hemos considerado algunos elementos que pueden tener relevancia en el deterioro de la función renal y que pueden actuar como factores de confusión, entre los 
que podemos señalar el consumo crónico de fármacos nefrolesivos, entre los que destacan indudablemente los AINE's.

Además de esto y considerando que hacemos referencia exclusivamente a pacientes hipertensos hubiera sido interesante una determinación más precisa de las cifras de tensión arterial. El cálculo de la presión media tras varias tomas en distintos días, la utilización de AMPA e incluso de en los casos en los que hubiera estado indicado el empleo de MAPA hubieran proporcionado un valor añadido a nuestro estudio.

En resumen, nuestro trabajo aporta fundamentalmente la confirmación de que también en los pacientes hipertensos seguidos en nuestra consulta no es desdeñable el porcentaje que presentan alteración de la función renal. Insistimos también en la conveniencia de valorar la función renal no solo mediante la Crp, apoyando la utilización sistemática de ecuaciones predictivas que nos permiten diagnosticar a un buen número de pacientes con ERC que se ocultan tras valores normales de Crp.

\section{BIBLIOGRAFIA}

1. Levey AS, Coresh J, Blak E, Kausz AT, Levin A, Steffes MW, et al. National Kidney Foundation Practice Guidelines for Chronic Kidney Disease: Evaluation, Clasification and Stratification. Ann Intern Med. 2003; 139: 137-147.

2. Coresh J, Astor BC, Greene T, Eknoyan G, Levey A. Prevalence of chronic kidney disease in the adult US population: Third national health and nutrition examination survey. Am J Kidney Dis. 2003; 41: 1-12.

3. Otero A, Abelleira A, Camba MJ, Pérez C, Armada E, Esteban J, Gayoso P. Prevalencia de insuficiencia renal oculta en la provincia de Orense. Nefrología. 2003: (Supl 6): abstract 26.

4. Simal F, Martin Escudero JC, Bellido J, Arzúa D, Mena FJ, González I, Alvarez AA, Tabuyo MB, Molina A. Prevalencia de la enfermedad renal crónica leve y moderada en población general. Estudio Hortega. Nefrología. 2004; 24: 329-337.
5. Almirall J, Vaqueiro M, Antón E, Baré ML, González V, Jaimez E et al. Prevalencia de la insuficiencia renal en la población general mayor de 64 años y episodios cardio-vasculares. Nefrología. 2005; 6: 655-662.

6. Fox CS, Larson MG, Leip EP, Culleton B, Wilson PWF, Levy D. Predictors of new-onset kidney disease in a community-based population. JAMA. 2004; 291: 844-850.

7. Hsu CY, Mc Culloch CE, Darbinian J et al. Elevated blood pressure and risk of end-stage renal disease in subjets whitout baseline kidney disease. Arch Intern Med. 2005; 165: 923-8.

8. Duncan L, Heathcote J, Djurdejv O, Levin A. Screening for renal disease using serum creatinine:who are we missing? Nephrol Dial Transplant. 2001; 16: 1042-6.

9. Fernandez-Fresnedo G, De Francisco ALM, Rodrigo E, Piñera C, Herraez I, Ruiz JC, et al. Insuficiencia renal "oculta" por valoración de la función renal mediante la creatinina sérica. Nefrología. 2002; 22: 144-51.

10. Cockroft DW, Gault MH. Prediction of creatinina clearance from serum creatinine. Nephron. 1976; 16(1): 31-41.

11. Vervoort G, Willems HL, Wetzels JFM. Assessment of glomerular filtration rate in healthy subjects and normalbuminuric diabetic patients:validaty of a new (MDRD) prediction equation. Nephrol Dial Transplant. 2002; 17: 1909-13.

12. National Kidney Foundation Kidney Disease Outcomes Quality Initiative (NKF K/DOQI) Am J Kidney Dis. 2002; 39 (Suppl 1): S1-S266.

13. Buitrago F, Calvo JI, Gómez-Gimenez C, Robles $\mathrm{N}$, Angulo E. Comparación y concordancia de las ecuaciones de estimación de filtrado glomerular de Cockroft-Gault y MDRD en el diagnóstico de enfermedad renal crónica oculta. Nefrología 2008; 28(3): 301-310.

14. De Francisco A, De la Cruz JJ, Cases A, De la Figuera M, Egocheaga MI, Górriz JI et al. Prevalencia de la insuficiencia renal en Centros de Atención Primaria en España: Estudio EROCAP. Nefrología 2007; 27: 311-312.

15. Pozuelos G, Molina L, Romero JJ, Diaz N, Cañón L, Buitrago F. Prevalencia de la insuficiencia renal oculta estimada mediante fórmulas de cálculo del grado de función renal en hipertensos mayores de 60 años, remitidos para medición ambulatoria de la presión arterial. Aten Primaria 2007; 39(5): 247-53. 
16. Alcázar R, Egocheaga Ma I, Orte L, Lobos J M⿻, González E, Älvarez F. Documento de consenso SEN-semFYC sobre la enfermedad renal crónica. Nefrología 2008; 28(3): 273-282.

17. Olivares J, Guillén F, Sánchez JJ, Morales-Olivas FJ. Effect of arterial preassure and age on renal function. The "Care for the Kidney" study. Nefrología 2003; 23(2): 137-44.

18. Molina A, Simal F, Martín JC, Bellido J, Ardúa D, Mena FJ et al. Prevalencia de la enfermedad renal crónica leve y moderada en la población general. Estudio Hortega. Nefrología 2004; 4: 329-337. 
